\title{
The hypothetical way of progress
}

Virginia A. Huszagh and Juan P. Infante

Biologists, and the journals that publish their papers, tend to dismiss theoretical work. Yet it is through ideas, not the mere generation of data, that the course of science is changed.

Is the hypothesis in the biological sciences an endangered species? The history of scientific thought shows that ideas are usually the driving force leading to new discoveries, not the accumulation or cataloguing of data. Many areas of biology, however, including biochemistry, seem stuck with nineteenth-century authoritarian ideology, which favours data production over generating new ideas or questioning stale paradigms. This encourages a kind of 'excellence in mediocrity', which drives off creative minds and attracts researchers who imitate rather than innovate'.

The importance of clearly formulated hypotheses which connect data in new ways is demonstrated by the Nobel prizewinning examples of Watson and Crick's hypothesis on DNA structure, Jerne's hypothesis on the origin of immunological diversity, and Mitchell's hypothesis on oxidative phosphorylation. Nevertheless, theoretical work in biology is often viewed as 'speculation'. This is not surprising considering that PhD students in the biological sciences receive little or no exposure to the history and philosophy of scientific thought, making the degree a misnomer. Few biologists can distinguish between speculation, demarcated hypotheses and theories. Even fewer appreciate the need for revolutionary hypotheses; and fewer still can generate them.

\section{Falsifiable predictions}

Understanding the fundamental differences among these concepts is essential if potentially productive proposals are to be distinguished from those that are blind alleys. A hypothesis, to qualify as scientific, must be both grounded (consistent with known experimental observations and fundamental principles) and demarcated (have necessary falsifiable predictions $)^{2-4}$; it thus enables meaningful, directed experimentation. A speculation, on the other hand, is an idea which might appear plausible or even inspiring, but which does not possess the necessary falsifiable predictions needed to make it fertile ground for experimentation. Various so-called 'hypotheses' in biology (often mistakenly labelled as 'theories', which are corroborated hypotheses or related sets of hypotheses) are no more than post hoc explanations or nonmechanistic mathematical formulations of data, whose necessary predictions have never been developed or tested. All too often such "black box"3 or pseudohypotheses are accepted more for sociological than for scientific reasons ${ }^{5,6}$.

Mitchell's chemiosmotic hypothesis of oxidative phosphorylation ${ }^{7}$ is a classic example of a true demarcated hypothesis in biochemistry. It contained only a reinterpretation of evidence already in the literature; it was based largely on theoretical principles ${ }^{8}$; and it proposed several necessary and specific falsifiable predictions. This hypothesis did not arise from a lucky accident, but resulted from an acute appreciation of the inadequacy of the prevailing paradigm, a critical analysis of the literature, and a good deal of reflection and imagination. Mitchell's theoretical work eventually revolutionized the field concerned, although he himself did only a small part of the experimental testing.

What allowed Mitchell to propose his ideas was not that he had access to any new data, but that his mind worked differently. Perhaps it is time that biologists recognized what physicists discovered early this century: that different minds are suited to different aspects of research, and science as a whole benefits by the interplay of theorists and experimentalists. A theorist's strength, and enjoyment, is in proposing testable ideas, whereas that of an experimentalist is in devising tests of such ideas. One cannot thrive without the other; the abilities needed for, and enjoyment derived from, each endeavour are different.

Except in a few isolated areas, those with abilities and a predisposition to do theoretical work find little opportunity or encouragement in biology. Biology is far behind physics in this regard. Indeed, biology is sometimes said to be too 'complex' to allow for general principles; such an attitude is encouraged by observationalists who generate and catalogue vast amounts of data without being able to propose coherent mechanistic explanations of them. This ideology also ignores the power of data that are inconsistent with accepted paradigms, because exceptions are merely seen as part of nature's complexity. The theorist, on the other hand, views inconsistencies as nature's way of saying we are missing something (perhaps the boat).

Only a few biology journals devote themselves largely to non-experimental work (of which hypotheses are only a subset), and they tend not to be widely read, particularly not by experimentalists. Those who review hypotheses in biology are usually data-generators who do not know the elements of importance and who thus make non-scientific sociologically based objections that are irrelevant to the construction or purpose of a hypothesis.

\section{Dismissive comments}

The theorist frequently encounters such dismissive comments as: "the author presents no new data"; "the author has not done the experiments himself"; or "the author is advised to do the experiments first and return after the hypothesis is "proven'". Those who take such an attitude are blind to the communal aspect of the scientific enterprise, that the person who proposes an idea need not be the one to test it. Where would physics be if Einstein had had to 'prove' his ideas before publishing them?

Several changes might allow for a more fruitful partnership between theorists and experimentalists in biology. First, more journals could encourage and publish hypotheses. Second, they could provide criteria to define the elements of a legitimate hypothesis. Referees of hypotheses might also be provided with these criteria, to encourage them to make genuine rather than spurious objections. But publication of more testable ideas will achieve little until experimentalists are disposed to seek them out rather than paddling in circles in their own little ponds - it took six years after he published them for Mitchell's ideas to be seriously tested by others. Of course, many so-called 'hypotheses' offer little of the mechanistic insight needed to induce experimentalists to test them; both sides of the equation need improvement. Finally, a few grants to encourage theoretical work and reflection in biology might be in order. After all, only ideas can make sense out of data; data alone do not generate ideas.

Virginia A. Huszagh and Juan P. Infante are at The Institute for Theoretical Biochemistry and Molecular Biology, PO Box 4512, Ithaca, New York 14852-4512, USA.

1. Lane. L.C. Nature 335, 109 (1988)

2. Popper, K.R. The Logic of Scientific Discovery 27-135 (Harper \& Row, New York, 1968).

3. Bunge, M. Scientific Research. 1. The Search for System 222-304 (Springer, New York, 1967)

4. Lakatos, 1. in Criticism and the Growth of Knowledge (eds Lakatos, I. \& Musgrave, A.) 91-196 (Cambridge University Press, 1970).

5. Kuhn, T.S. The Structure of Scientific Revolutions (2nd edn) 1-210 (University of Chicago Press, 1970)

6. Ninio, J. Trends biochem. Sci. 6, VII-1X (1981).

7. Mitchell, P. Nature 191, 144-148 (1961).

8. Mitchell. P. A. Rev. Biochem. 46, 996-1005 (1977). 